Encyclopedia of Health Communication

\title{
Nonverbal Communication in Health Settings
}

Nonverbal communication is communication without words. Although the interest for caregiver and patient nonverbal communication is relatively young, studies on nonverbal behavior in the realm of caregiver-patient interactions have yielded many interesting results during the last decades. First in correlational designs and then in experimental ones, researchers have shown that healthcare providers' nonverbal communication predicts several important patient outcomes. For instance, we know that affiliative nonverbal behaviors such as smiling, nodding, or leaning forward increase patient satisfaction, trust, and adherence. Moreover, we begin to gain insight into how patient individual characteristics shape their reactions to a given caregiver's communication style. Researchers have not only investigated caregivers' nonverbal behavior but have also looked at patients' nonverbal behavior. In the psychotherapeutic context, in particular, patients' nonverbal behavior can contain important information about the patient's disease (e.g., depressive disorder, schizophrenia, autistic troubles). Also, enhancing patients' nonverbal skills defined as correctly reading and interpreting others' nonverbal behaviors, as well as expressing oneself nonverbally in a way that is congruent with one's communicative goals can be part of the therapy. As the field of caregiver-patient communication developed, both theoretically and empirically, we have gained more knowledge about how physicians, nurses, therapists, and other healthcare providers should best communicate with their patients, interpret their patients' nonverbal behavior, and adapt to their patients' nonverbal behavior in order to ensure best possible care.

\section{Definition, types, and functions of nonverbal behaviors}

Nonverbal communication includes facial, postural, gestural, and vocal (paralinguistic) cues. Nonverbal cues serve different purposes, such as signaling personal characteristics (e.g., extraversion, intelligence) and interpersonal orientations and goals (e.g., interest, attention, antipathy, intimacy, or status), coordinating verbal speech, and/or expressing emotions. Face cues include, for instance, gazing, smiling, frowning, eyebrow raising, and general facial expressivity. Postural cues are, for instance, arm and leg positions and movements, body orientation, and interpersonal distance. Gestures can be emblematic when having a defined linguistic content (e.g., saying no by doing repetitive left-right movements of the forefinger), illustrative when gesturally expressing a concept or an object (e.g., indicating the size of a box with the hands), regulative when facilitating verbal exchanges (e.g., indicating with a movement of the hand that the interaction partner should continue to talk), adaptive when responding to a personal need (e.g., scratching one's itchy head), or emotional when conveying an emotion (e.g., clenching one's fist when feeling 
irritated). Vocal cues include, as examples, loudness of the voice, voice pitch, speech rate, and voice tone variations.

Nonverbal communication can complement, repeat, reinforce, substitute, regulate, or contradict verbal communication. When there is contradiction between the two communicative channels, it seems that people rely more on nonverbal cues than on verbal cues when trying to infer meaning from the communication. For instance, when someone says "I like you" with a stoned expression that conveys the opposite message, people will typically form their judgment of the other person's liking by relying on the facial cues and deduce that the person is not serious or honest when saying that he or she likes them. A common explanation for the preference given to nonverbal cues when decoding others' messages is that nonverbal behaviors are under less of a conscious control than verbal behaviors. As a consequence, people would tend to consider nonverbal cues as more difficult to fake and thus as more indicative of their interaction partners' real intentions and/or states.

\section{Caregivers' nonverbal behavior}

In the caregiver-patient relation, two aspects of nonverbal behaviors are commonly studied: affiliative behaviors and controlling behaviors. Affiliative nonverbal behaviors encompass all nonverbal behaviors that aim at creating a positive relationship between the caregiver and the patient by communicating attention, interest, empathy, and/or friendliness. They include, for instance, reinforcing behaviors such as smiling and nodding, as well as signs of involvement such as facial expressiveness, facing body posture, and forward leaning. Controlling nonverbal behaviors are all nonverbal behaviors that aim at gaining or maintaining control over the interaction partner. They include, for instance, talking in a loud voice or adopting an expansive body posture (i.e., occupying a maximum of space).

Affiliative nonverbal behaviors in the caregivers have been shown to positively influence patient outcomes. Experimental studies suggest that when caregivers adopt affiliative nonverbal behaviors such as doing moderate to high eye contact with the patient, rarely looking at the patient chart, using concerned facial expressions, smiling frequently, leaning forward, and sitting relatively close (two feet) to the patients, patients are more satisfied with their caregivers, trust them more, and recall more medical information than when caregivers adopt less affiliative behaviors (e.g., little eye contact, frequent looking at the chart, neutral facial expressions, leaning backward, and sitting four feet away from the patient). In the same way, when physicians touch their patients gently on the forearm for one to two seconds at the end of the visit while stressing the importance of following the medication, patients were more likely to actually take their medication as prescribed (i.e., they showed more medication adherence) than when the physician stressed the importance of following the medication without touching the patients. 
Correlational research has revealed many associations between caregivers' affiliative behaviors, as expressed nonverbally, that replicate the results of experimental studies in real medical settings. For instance, meta-analytical results show that physicians' and nurses' affiliativeness (warmth) and listening to the patient are associated with more patient satisfaction. Correlational research also suggests associations between caregivers' nonverbal affiliativeness and patient outcomes that have not (yet) been shown by experimental research. For instance, caregiver's gazing at the patient has been related to more successfully identifying his or her psychological distress. Caregivers' expressiveness (operationalized differently across studies, e.g., through more nodding, more smiling, more frowning, more gestures, and/or more variations in the tone of voice) is related to more patient satisfaction in patients of different ages, as well as to better physical and cognitive functioning in elderly patients.

Research on caregivers' controlling nonverbal behaviors (e.g., visual dominance through looking at the patient while talking to him or her and not looking at the patient while listening to him or her, expressivity, close interpersonal distance, loud tone of voice, postural expansiveness) is comparatively scarce, and mostly correlational, but it shows that those behaviors are generally associated with less patient satisfaction. Also, surgeons whose tone of voice is more dominant have more malpractice claims. In particular, patients seem to dislike controlling nonverbal behaviors when such behaviors are expressed by female caregivers - probably because controlling behaviors contradict the female gender role stereotypes. Conversely, patients seem to show more satisfaction when male physicians show more controlling behaviors such as talking with a louder voice and showing postural expansiveness, probably because controlling behaviors are in accordance with the male gender role stereotypes.

Despite the fact that the above mentioned findings hold true on average, a more finegrained view on the type and intensity of caregiver nonverbal behavior - as well as on the characteristics of the caregiver and of the patient - is in order. The effect of caregivers' nonverbal behaviors on patient outcome sometimes depends of the exact form of the given nonverbal behavior. For instance, soft touch increases patient adherence, while touch perceived as dominant or controlling seems to have a negative effect on patient outcomes; the effect of physician touch thus probably depends on the kind of touch (slight tactile contact vs. brusque or invasive contact). Moreover, the rule 'the more the better' cannot systematically be applied to physician nonverbal behaviors shown to share positive associations with patient outcomes. For many behaviors, moderation seems better than extremes. For instance, too much or too little of physician gazing seem equally detrimental to patient satisfaction, and moderate levels of gazing are related to the highest satisfaction rates. And, caregivers' and patients' individual characteristics (e.g., personality, attitude, gender, age, educational level) might moderate their reactions to caregivers' degree of affiliation and control. For instance, patients who are anxious (as judged from their voice by independent raters) are more tolerant, or even prefer, physicians who sound angry than 
patients who are less anxious. Also, nonverbal behavior might have different effects on patient outcomes depending on the type of healthcare provider who expresses it. Metaanalytical results show, for instance, that physicians' negativity (e.g., expressed anger or hostility) is not related to patient satisfaction, whereas nurses' negativity is. Even if we lack, at this stage, clear explanations for such differences in influence of caregivers' nonverbal communication on patient outcomes depending on the caregivers and on the patients, such effects are documented and need to be taken into account.

\section{Patients' nonverbal behavior}

Patients' nonverbal behavior is influenced by physicians' nonverbal behaviors. Research shows for instance that patients tend to mirror affiliative behaviors in the physician (e.g., gazing, facing orientation) and that interactional synchrony between the physician and the patient with regard to nonverbal behavior (i.e., when physician and patients display similar behavior at the same time) is associated with more interactional comfort (i.e., patients and physicians feel more at ease). In the psychotherapeutic domain as well, therapists are perceived more positively by patients when therapists and patients mirror each other's body movement and posture than when they do not. This might be explained by the fact that mirroring is associated with greater perceived rapport and liking between the interaction partners.

Nonverbal sensitivity (defined as correctly inferring others' traits and states on the basis of their nonverbal behavior) is an important skill for physicians to possess. Physicians might lack nonverbal sensitivity when it comes to recognizing affective states in their patients or to evaluating patient satisfaction and liking of the physician. Female medical students show more nonverbal sensitivity than male medical students. Lack of nonverbal sensitivity in physicians is potentially a problem, notably because this skill is associated with more patient satisfaction and better appointment keeping in the patients.

Nonverbal sensitivity is important in the psychotherapeutic domain as well. Patient nonverbal behaviors can be used for diagnosis by therapists because nonverbal characteristics accompany most psychopathologies (e.g., mood disorders, schizophrenia, autism, substance dependency). Individuals suffering from depression or from schizophrenia typically show reduced emotional expressiveness (e.g., non-expressive facial displays, gaze aversion, scarcity of gestures, monotonous voice tone). Also, such patients have a poorer ability to correctly infer others' states (e.g., emotions) on the basis of others' nonverbal behavior (i.e., less nonverbal sensitivity) compared to healthy individuals. Moreover, patients' nonverbal behavior can provide information about the patient to the therapist that the patient is not willing or able to give verbally (e.g., distress, deception). Finally, nonverbal training can be part of the therapy, for instance when patients with nonverbal deficits (e.g., autistic patients who typically have difficulties in understanding others' emotion) are trained to encode and decode nonverbal behavior in order to enhance their social skills. 


\section{Methods and perspectives}

Most research in the field of caregiver-patient communication is correlational. This typically ensures high external validity (good potential for generalizability of the findings) but does not allow for causal inferences and sometimes poses problems of interpretation regarding the direction of the discovered relation. For instance, an association between physician nonverbal expressivity and patient positive health outcomes can be interpreted in different ways: 1) physician expressivity might have positively influenced patient health outcomes, or 2) physicians might have been more expressive with patients who were in better health, or 3) a third variable (e.g., the weather) might have influenced both physician expressivity and patient health status. This is why other researchers have built experimental design. These designs, by manipulating physician nonverbal behavior and maintaining a high level of control over potential confounding variables, have enabled researchers to establish causal association (e.g., between physician nodding and patient satisfaction). However, experimental designs in the field of caregiver-patient interactions have often relied on socalled analogue patients (healthy participants asked to put themselves into the shoes of real medical patients) and/or on actors playing the role of physicians (i.e., simulated patients) and are more likely to suffer from low external validity (limited potential for generalizability of the findings). Some researchers have attempted to go beyond the respective limitations of correlational field studies and experimental analogue studies by running experimental field studies (i.e., studies using experimental designs but in real medical settings). However, such attempts are still scarce and will need to be developed in the future.

Researchers in the field of caregiver-patient communication sometimes lack consensus regarding the terminology. Very similar but not completely overlapping constructs (e.g., affiliativeness, warmth, communion, friendliness, immediacy) are typically used in an interchangeable way, which makes comparisons between studies difficult. Also, the same constructs are not always operationalized in the same way by different researchers. Some attempts have been made to put order in the field, such as proposing to apply the principle of the interpersonal theory to the field of caregiver-patient interaction and to organize past research along two main dimensions: the dimension of affiliation (which encompasses all verbal and nonverbal behaviors going from extremely hostile to extremely friendly) and the dimension of control (which encompasses all verbal and nonverbal behaviors going from extremely submissive to extremely dominant). Future will tell us if such organizing framework and terminology will actually be adopted by researchers in the field.

The field of caregiver-patient communication is sometimes criticized for not building or relying enough on theory. Initiators of the criticism point out that few theories exist in the field, and indeed - with a few exceptions such as the typology of physician-patient relationships or the E4 model for physician-patient communication - very little theories exist that were built in the field and exclusively focus on caregiver-patient interactions. Many 
theories exist within the broader field of communication (e.g., the parallel process model of nonverbal communication, the equilibrium theory, the discrepancy arousal theory, the interaction adaptation theory), but they are sometimes too general for making predictions within the specific context of caregiver-patient interactions.

Despite those current limitations, the study of nonverbal communication in healthcare settings has considerably enriched the domain of caregiver-patient interactions. We now have evidence that nonverbal behavior plays a key role during medical and psychotherapeutic interviews. As research shows, enhancing caregivers' nonverbal communication and gaining knowledge about both caregivers' and patients' nonverbal cues can enhance the quality of care as a whole.

Gaëtan Cousin \& Marianne Schmid Mast

University of Neuchatel, Switzerland

See Also: Adherence to medical regimens; Doctor-Patient Communication; Interpersonal Communication Skills; Patient/relationship-centered communication/medicine; Satisfaction as related to communication

Futher Readings:

Beck, R. S., Daughtridge, R., \& Sloane, P. D. (2002). Physician-patient communication in the primary care office: A systematic review. The Journal of the American Board of Family Practice, 15, 25-38.

Hall, J. A. (2011). Clinicians' accuracy in perceiving patients: Its relevance for clinical practice and a narrative review of methods and correlates. Patient Education and Counseling, 84, 319-324.

Mead, N., \& Bower, P. (2002). Patient-centered communication and outcomes in primary care: A review of the literature. Patient Education and Counseling, 48, 51-61.

Robinson, J. D. (2006). Nonverbal communication and physician-patient interaction. In V. Manusov \& M. L. Patterson (Eds.), The Sage Handbook of Nonverbal Communication. Thousand Oaks, CA: Sage Publications.

Schmid Mast, M., Hall, J. A., Klöckner, C., \& Choi, E. (2008). Physician gender affects how physician nonverbal behavior is related to patient satisfaction. Medical Care, 46, 1212-1218. 